\title{
DATA MINING FOR ENERGY MANAGEMENT IN A WATER DISTRIBUTION NETWORK
}

\author{
Hohyun Lee, Dae Wook Kim, Sung Taek Hong and Gang Wook Shin \\ K-water, Daejeon, Korea
}

\begin{abstract}
In the water treatment process, it is important to predict the flow used by citizens and control valves for using large reservoir tanks maximally. In this study, flow predictions are proposed by applying several non-linear algorithms with recursive form to minimize additional learning. To control the valves properly, the target levels of each tank are determined based on flow prediction and genetic algorithms. A simple polynomial learning algorithm is also proposed to control the valves less frequently than a conventional feedback controller until the target tank levels can be reached, which also help not to break down the valves. The proposed method to maximize the usage of reservoir tanks is expected to reduce the electric fee and improve water quality.
\end{abstract}

\section{KEYWORDS}

Water, Reservoir Tank, Learning Algorithm, Electric Fee

\section{INTRODUCTION}

Unlike electricity, water can be stored in large tanks for more than a dozen hours and citizen's water demands have certain patterns which may be estimated by statistical approaches.

Most of the costs of sending treated water to citizens are attributed to electrical fees which are caused by pumping (Reynolds, 2010). The electric fee depends on the time and season, which is called a load shift. The policy was even applied to public water suppliers. In addition, a new optimization target, the on/off minimization of pumps, should be achieved not to degrade water quality. If input flow is suddenly increased to water treatment plants by the use of more pumps, more turbidity and particles will be supplied to the citizens, which can be minimized by the on/off minimization algorithm.

The optimization of both load shift and on/off minimization are based on the control level of the storage tanks, which needs to be controlled by their electric valves. But it is not easy to control because of a variety of constraints and non-linearity.

\section{PROPOSED ALGORITHM}

In statistics, predictions are possible about unknown parameters, when the number of measurements are larger than the number of unknown parameters and the measurement errors are normally distributed. The database for water treatment processing has already accumulated massive amounts of data to be analyzed. It is enough to estimate water data with non-linearity and time series. Many algorithms have been introduced and reviewed to estimate the short-term and long-term demands.

Multi-Layer Perceptron and adaptive neuro fuzzy inference systems are used frequently. While a single layer perceptron network cannot solve nonlinear problems, a multi-layer perceptron network gives a solution to the problems (Alpaydin, 2004). Figure 1 (a) describes the 3 layers of the perceptron to approximate flow demand, in which the estimation error is updated by a back-propagation algorithm (Rumelhart, Hinton, \& Williams, 1985).

ANFIS stands for adaptive neuro fuzzy inference system, which was first proposed by Roger Jang. It can identify nonlinear components linearly in a control system and predict times series (Jang, 1993). The architecture of ANFIS is shown in Figure 1 (b). 


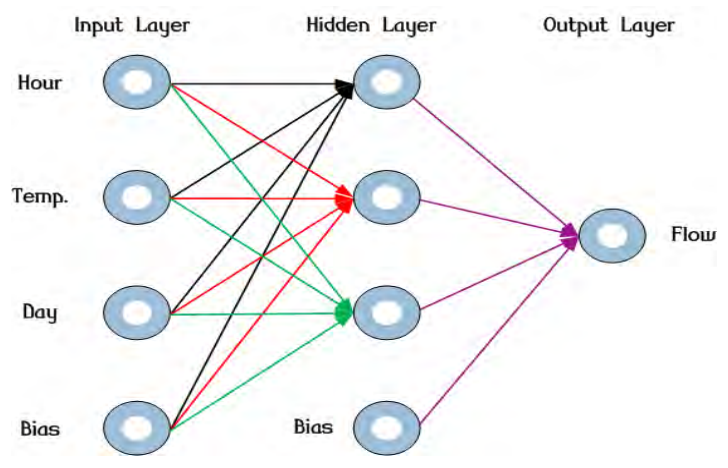

(a)

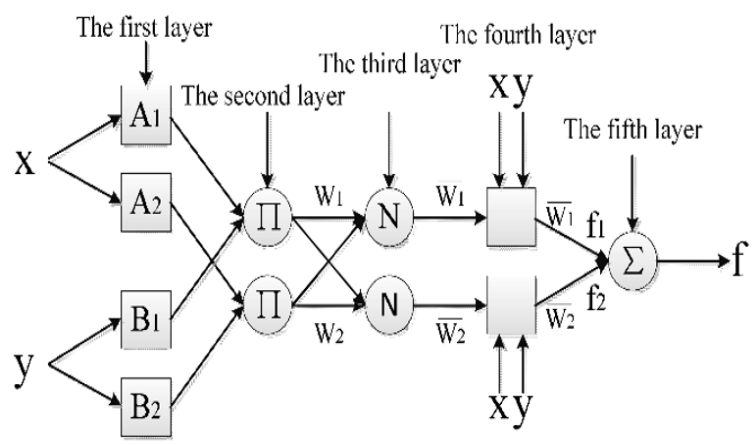

(b)

Figure 1. Structure of a learning algorithm: (a) Basic structure of MLP, (b) Basic structure of ANFIS

\section{EXPERIMENT}

Flow prediction and optimal pump control are necessary to have all of the information of tank levels and flows, which also help to control electric valves. But the data and controlling valves are restricted to some of the large tanks. Prediction and control might be achieved under these conditions. At first, the main large tanks flow are estimated by ANFIS, and then the estimated flow will extend to all of tanks. The flow is compensated by a recursive algorithm that calculates the error between the estimated flow and real output for 24 hours. Then, the relation between valve position and flow can also be approximated by the polynomial regression algorithm as shown in Figure 2.

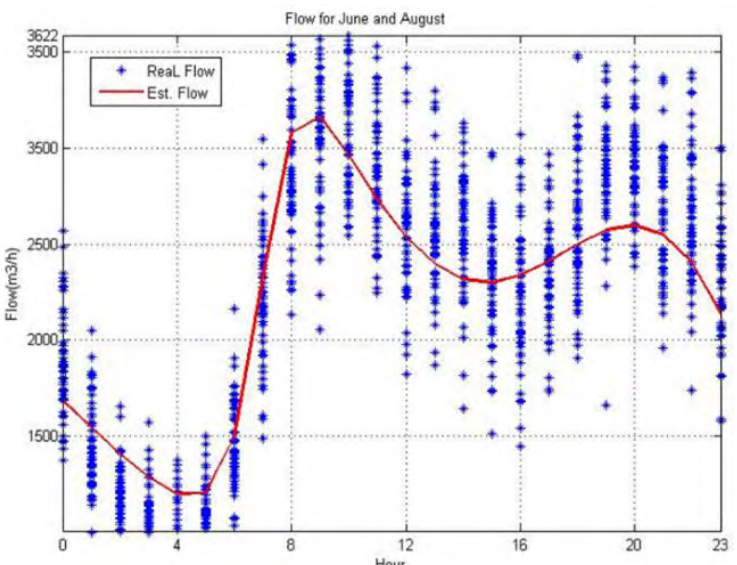

(a)

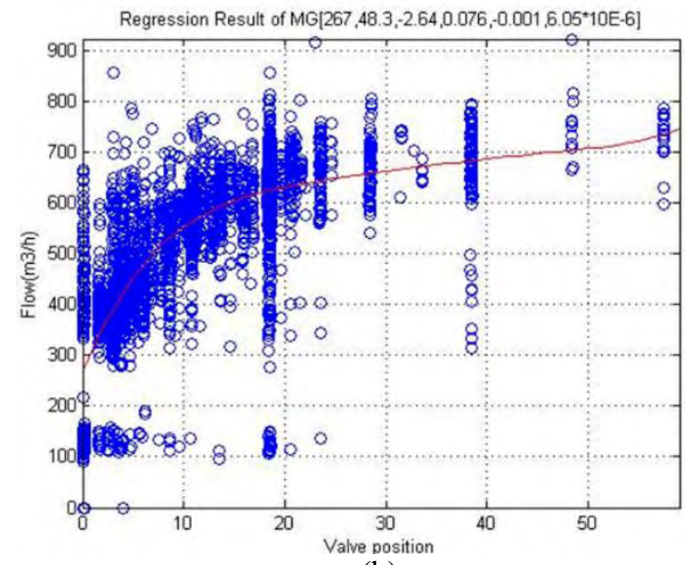

(b)

Figure 2. (a) Flow prediction by ANFIS, (b) Basic structure of ANFIS

General algorithms such as regression, neural network and support vector machine are considered and their results are shown in Table 1. LR(Linear Regression) had the worst result, which means hourly flow doesn't have a linear trend. Other algorithms had almost the same results.

Table 1. Estimation result by each algorithm for flow prediction

\begin{tabular}{c|c|c|c|c|c|c|c}
\hline \multicolumn{2}{c|}{ Error } & LR & Polynomial & $\begin{array}{c}\text { MLP } \\
\text { (Hidden 3) }\end{array}$ & $\begin{array}{c}\text { MLP } \\
\text { (Hidden 20) }\end{array}$ & ANFIS & LS_SVM \\
\hline \multirow{2}{*}{$\begin{array}{c}\text { Train } \\
\text { Error }\end{array}$} & MAPE & 22.75 & 13.47 & 13.28 & 12.49 & 12.80 & 12.22 \\
\cline { 2 - 8 } & MSE & 178,050 & 77,787 & 78,993 & 68,841 & 71,856 & 67,186 \\
\hline \multirow{2}{*}{$\begin{array}{c}\text { Test } \\
\text { Error }\end{array}$} & MAPE & 22.67 & 13.72 & 13.42 & 12.87 & 12.95 & 12.54 \\
\cline { 2 - 9 } & MSE & 179,070 & 80,486 & 81,202 & 72,572 & 74,107 & 71,052 \\
\hline \multicolumn{2}{l}{ \# of Parameter } & 4 & 12 & $\begin{array}{c}4 * 3+4 * 1 \\
=16\end{array}$ & $\begin{array}{c}4 * 20+21 * 1 \\
=101\end{array}$ & $\begin{array}{c}14 * 4 \\
=56\end{array}$ & $\begin{array}{c}18950 \\
(\text { SV }\end{array}$ \\
\hline
\end{tabular}


To control the valve, it should be decided to divide the target water flow to each tank. Supply water from WTP would be multiplied by each tank weight, which is calculated by the mean supply of water from each tank. Then, each flow is added or subtracted according to present and target levels.

$$
\begin{gathered}
\mathrm{F}_{i}^{\text {target }}=F^{\text {tankin }} \times \omega_{i}^{f}+Q_{i}^{\text {level }}=F^{i n} \times \omega_{i}^{f}+\left(L_{i}^{\text {target }}-L_{i}^{\text {present }}\right) \times Q_{i}^{\text {unit }} \\
\text { Where } \omega_{i}^{f}=\frac{u_{i}^{f}}{\sum_{i}^{f} u_{i}^{f}}, u_{i}^{f}=\frac{1}{n} \sum_{j=1}^{n} F_{j}^{\text {tankout }}, \\
F^{\text {takin }}=F^{\text {wtp }} \times \alpha+\Delta F_{i-1}^{w t p}, \Delta F_{i-1}^{w t p}=\Delta F_{i-2}^{\text {wtp }}+\left(F_{i-1}^{\text {wtp }}-F_{i-1}^{\text {truewtp }}\right)
\end{gathered}
$$

Because their flows are bounded in certain areas, they should be saturated in minimum and maximum flow as follow.

$$
\mathrm{F}_{i}^{\text {target }}=\max \left(F_{i}^{\text {min }}, F_{i}^{\text {target }}\right) \text { and } \mathrm{F}_{i}^{\text {target }}=\min \left(F_{i}^{\text {max }}, F_{i}^{\text {target }}\right)
$$

If more than one of tank flow are over the limit, their burden must move to other spacious tanks as below.

$$
\Delta F^{\text {tank }}=F^{\text {tankin }}-\sum F_{i}^{\text {target }}, \rightarrow \text { Divide } \Delta F^{\text {tank }} \text { to spacious tanks }
$$

After the target flows are decided as above, the proposed valve controller should be working. Figure 3 shows that the target levels were reached.
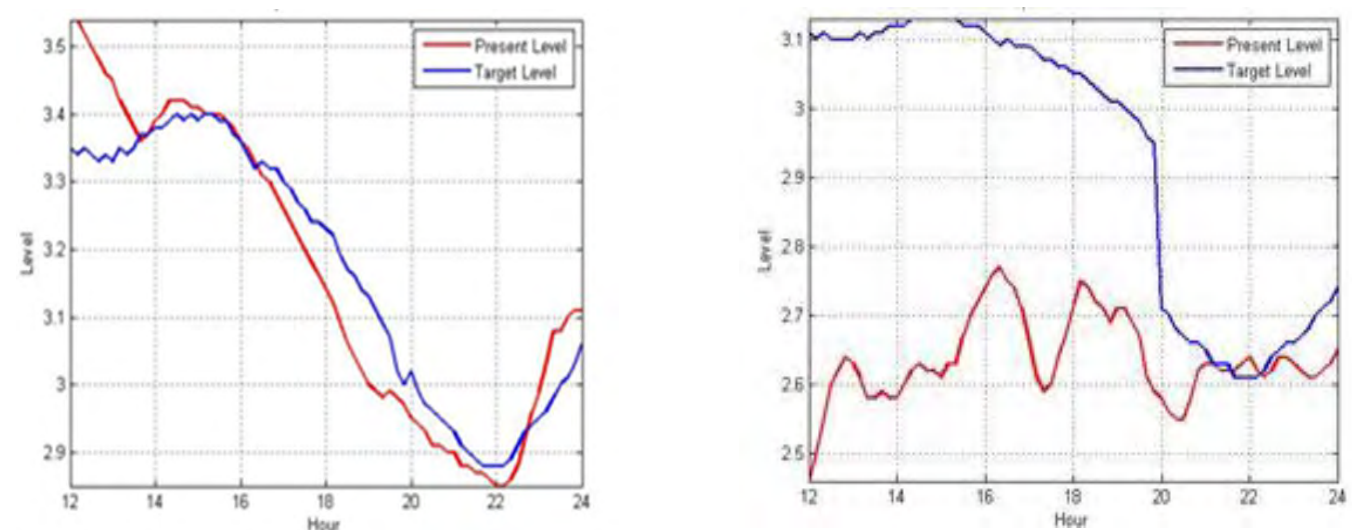

(a)

Figure 3. (a) Error comparison of JW tank level, (b) Error comparison of JM tank level

\section{CONCLUSION}

Flow predictions are estimated by learning algorithms with recursive form, which makes it possible to track the latest trends and it is not necessary to do any additional learning differently from existing methods. Then, optimal operation levels are determined to minimize costs based on flow prediction and genetic algorithm. The electric cost is different in a specific time and season, in which the tanks levels are estimated by the optimal algorithm. The number of operation pumps are calculated for 48 hours, by which target levels of each tanks are calculated according to load shift. While the target levels should be reached to reduce the operation costs, it was not easy to control the valves because there are many constraints such as low to high flow, level, and long operation intervals, which makes a normal PID controller useless. After modeled by simple polynomial learning algorithm, valves are controlled to use the least amount of time as possible and the constant level target in the WTP and equal distribution in the tanks can be reached. It is expected to maximize the usage of reservoir tanks, which is thought to reduce the electric fee and improve water quality.

\section{REFERENCES}

Alpaydin, E. (2004). "Introduction to machine learning", The MIT Press, 229-251

Jang, J.-S. R. (1993), ANFIS: Adaptive-Network-Based Fuzzy Inference System, IEEE Transactions on System, Man and Cybernetics, 23(3), 665-685

Reynolds, L. K. (2010). Improving energy efficiency of pumping systems through real-time scheduling systems. Integrating Water System-Boxall and Maksimovic(eds) 2010 Taylor and Francis Group, London, ISBN 978-0-415-54851-9

Rumelhart,D. E., Hinton, G. E., \& Williams, R.J. (1985). Learning internal representations by error propagation, Cognitive science, 4-8. 